Article

\title{
RFID RSS Fingerprinting System for Wearable Human Activity Recognition
}

\author{
Wafa Shuaieb ${ }^{1,2}$, George Oguntala ${ }^{1, * \mathbb{C}}$, Ali AlAbdullah ${ }^{1}$, Huthaifa Obeidat ${ }^{3}{ }^{\mathbb{D}}$, Rameez Asif ${ }^{1}$, \\ Raed A. Abd-Alhameed ${ }^{1,4}{ }^{\mathbb{D}}$, Mohammed S. Bin-Melha ${ }^{1}$ and Chakib Kara-Zaïtri ${ }^{5}$ \\ 1 Department of Biomedical and Electronics Engineering, Faculty of Engineering and Informatics, University \\ of Bradford, Bradford BD7 1DP, UK; w.s.a.shuaieb@student.bradford.ac.uk (W.S.); \\ a.a.alabdullah1@bradford.ac.uk (A.A.); r.asif1@bradford.ac.uk (R.A.); r.a.a.abd@bradford.ac.uk (R.A.A.-A.); \\ m.b.melha@bradford.ac.uk (M.S.B.-M.) \\ 2 Faculty of Engineering, Omar AL-Mukhtar University, El-Beida, P.O. Box 919, Libya \\ 3 Faculty of Engineering, Jerash University, Jerash 26150, Jordan; h.obeidat@jpu.edu.jo \\ 4 Basra University College of Science and Technology, Basra 61004, Iraq \\ 5 Department of Mechanical and Energy Systems Engineering, Faculty of Engineering and Informatics, \\ University of Bradford, Bradford BD7 1DP, UK; c.karazaitri@bradford.ac.uk \\ * Correspondence: G.A.Oguntala@bradford.ac.uk
}

Received: 9 January 2020; Accepted: 10 February 2020; Published: 12 February 2020

check for updates

\begin{abstract}
Alternative healthcare solutions have been identified as a viable approach to ameliorate the increasing demand for telehealth and prompt healthcare delivery. Moreover, indoor ocalization using different technologies and approaches have greatly contributed to alternative healthcare solutions. In this paper, a cost-effective, radio frequency identification (RFID)-based indoor location system that employs received signal strength (RSS) information of passive RFID tags is presented. The proposed system uses RFID tags placed at different positions on the target body. The mapping of the analysed data against a set of reference position datasets is used to accurately track the vertical and horizontal positioning of a patient within a confined space in real-time. The Euclidean distance model achieves an accuracy of $98 \%$ for all sampled activities. However, the accuracy of the activity recognition algorithm performs below the threshold performance for walking and standing, which is due to similarities in the target height, weight and body density for both activities. The obtained results from the proposed system indicate significant potentials to provide reliable health measurement tool for patients at risk.
\end{abstract}

Keywords: fingerprinting; human activity recognition; patient tracking; indoor ocalization; RFID

\section{Introduction}

Recent time has witnessed increasing cases of longevity as humans live longer than ever. This is due to the various breakthroughs in medicine, biomedical engineering and wireless communication, which subsequently results in improved health services. The United Nations (UN) report show that the number of the elderly and infants across the world is growing and is expected to double by the end of 2050. However, the number of chronic and degenerative diseases in both the elderly and young people is on the rise and results in the increasing cases of disability [1,2]. These degenerative diseases including Alzheimer, Dementia, Parkinson, Osteoporosis and incontinence affects the cognitive capabilities of the humans, making them disabled and sometimes vulnerable and in need of constant support, making the demand on improved healthcare and service delivery critical [3].

Alternative healthcare solutions have been identified as a viable approach to ameliorate the increasing, unsustainable demand for healthcare service delivery $[4,5]$. Alternative health solution 
serves as a precautionary measure to provide early warnings to various targets including patients, elderly and vulnerable. A common alternative health solution is the patient tracking system. Patient tracking is a key indoor ocalization application and provides valuable insight into a target health condition. For effective patient tracking, the target position and orientation are estimated using semantic information from different sensors to deduce the physical condition of the patient. Data sensing for target tracking is achieved using the wearable and ambient approach [6,7]. The wearable approach employs the use of sensors worn as wearable devices such as health and fitness wearable or embedded into household wares including furniture, toilet sits, etc. However, in ambient data sensing, sensors are integrated into the target's environment. The readings from the sensors are collected, interpreted for possible patient tracking using various data analytics algorithms including machine learning, neural network and deep learning [8,9]. The key aim of patient tracking is change detection by identifying changes in metric which represents a change point in time-series data within an indoor environment [10]. The effective manipulation of these metrics using a robust algorithm would assist in determining the shift in position and orientation of the target. However, accurate indoor target detection and tracking systems require overcoming the multi-path effect which is often a major propagation challenge in indoor environments. Additionally, the dynamic nature of human activities including human movement; such as walking, standing and posture which includes fall, lying down and sitting, makes target location detection and tracking an interesting research area.

With the emergence of the Internet of Things, indoor ocalization, especially in target tracking and monitoring using different indoor methods, devices and computational algorithms, is actively receiving intense attention in the literature [11,12]. Different authors have developed various innovative indoor systems to achieve target monitoring under different real-time conditions. Wan et al. employed high-resolution direction of arrival to effectively track the position of a patient [13]. The developed approach exhibits high tracking accuracy and performance over the traditional MUSIC algorithm. However, the proposed system becomes unreliable in the presence of non-line-of-sight (NLOS). Khoshhal et al. and Yin et al. employed the unsupervised approach to monitoring the daily activities of patients using the target locomotion pattern. In these studies, the locomotion pattern is used in finding the routine cluster at different time intervals within the room to understand associated human reactions and behaviours [14,15]. Bustamante et al. proposed a smart monitoring bed system for detecting fall in elderly people. The proposed system determines anomaly in the patient position to determine fall. However, the performance of the system suffers from false alarm with $35 \%$ of the alarm being false [16]. Motoi et al. proposed an unobtrusive e-health monitoring system using several monitoring devices and sensors for monitoring a patient cardiovascular disease during sleep [17]. Alahmadi et al. proposed a smart e-health monitoring system architecture capable of monitoring, ocalizat and sharing mobile patient data with medical experts [18]. Reilent et al. proposed a monitoring system for acquiring the medical and contextual data of the patient's automated home [19]. Kyriazakos et al. proposed an e-inclusive health system to track patients with mild dementia [20]. Ayase et al., Long et al. and Cuppens et al. employed the video-based approach to track the vital signs including heart measurement rate based on facial analysis, home fitness exercise and sleep monitoring and physical activity of patients [21-23]. Lawrence et al. explored the use of modern interactive games as a means to track and improve the physical and mental health of elderly patients [24]. Furthermore, the development of smart home as a means to track the physical and health condition of patients has also been explored. Oguntala et al. proposed the ambient human activity recognition approach using passive radio frequency identification (RFID) tags to unobtrusively sampled target activities [25,26]. Heikkel et al. proposed an intelligent furniture network for human posture and activity detection [27]. Freitas et al. proposed and implemented a monitoring system of a smart home system for disabled people, including those with visual and hearing impairments, activating a warning against indoor accidents using mobiles phones and a wireless sensor network [28]. Booranrom et al. proposed a smart bedroom, which monitors the daily activities for the elderly using Kinect, a camera which can detect a human body and voice movements and warns the elderly before falling out bed by controlling the 
electric devices in a timely manner [29]. Nevertheless, with the continuous demand for high return on investment on technological systems, the development of a simple, cost-effective wearable approach for reliable patient tracking becomes a critical design requisite. In the present work, a cost-effective target tracking system useful for monitoring the elderly, vulnerable and persons with disability is proposed.

The proposed methodology is based on processing data received from low-cost RFID tags mounted on ordinary clothing using the radio frequency fingerprinting technique. Radio fingerprinting using different indoor and outdoor technologies has been used for many applications including broadcasting, location-based applications, high precision applications and activity recognition [30-34]. However, to the best of the authors' knowledge, the proposed system would be first that uses RFID fingerprinting methodology for inferring the patient position and orientation in real-time. The objective of the present work is to develop a cost-effective, daily human activity recognition using the RSS fingerprinting method. The proposed approach serves as an efficient automated alarming system in nursing homes, as patient inactivity or stationary position over an extended period of time would trigger the attention of nursing staffs.

The paper is structured as follows: In Section 2, a detailed description of the methodology employed in the present work and the formulation of the received signal strength indicator (RSSI) model are presented. In Section 3, the performance of the Euclidean distance algorithm is evaluated using different performance metrics for all sampled activities of daily living (ADL). Section 4 concludes the findings of the present study.

\section{RSSI Model}

Target ocalization and activity recognition involve wearable data acquisition using the power distribution of passive RFID tags.

\subsection{Experimentation}

Six volunteers of varying height, weight and body density were selected for activity sampling.

The experiment was performed in the enclosed Sensor Design, Antenna and Propagation and Radio Frequency laboratory of the Faculty of Engineering and Informatics, University of Bradford. Different physical materials including furniture were placed within the activity sensing perimeter of $5 \times 5 \mathrm{~m}^{2}$ to simulate real-world scenario and achieve effective detection range. Moreover, two circularly-polarised Alien RFID readers operating at the European UHF of $866 \mathrm{MHz}$ with $5.5 \mathrm{dBm}$ gain were used to read the RFID tags. The RFID reader antennas were placed at a maximum height of $2.34 \mathrm{~m}$ to achieve maximum detection and resolution. Fifty-six lightweight, commercially-available passive RFID tag of dimensional geometry $74 \mathrm{~mm} \times 21 \mathrm{~mm}$ and a read range of $6 \mathrm{~m}$ with known ID_value was attached to seven distinct positions on the target. Each distinct tag location on the target was monitored by a set of eight tags as illustrated in Figure 1. Different physical objects to simulate real-world object occlusion were placed within a few metres of the target. Table 1 highlights the seven tag locations selected on the target. The seven distinct tag locations were selected since these locations were associated with various activities of daily living.

Table 1. Distribution of tag placement on the target.

\begin{tabular}{cc}
\hline Tag Location & Position on Target \\
\hline Location_1 & Head \\
Location_2 & Arm \\
Location_3 & Waist \\
Location_4 & Back \\
Location_5 & Thigh \\
Location_6 & Knee \\
Location_7 & Ankle \\
\hline
\end{tabular}




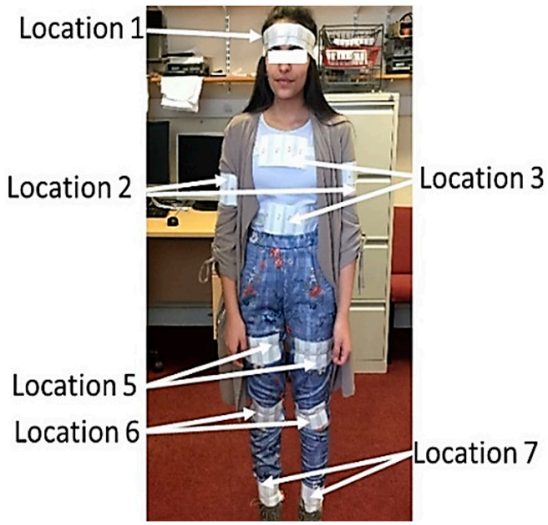

(a)

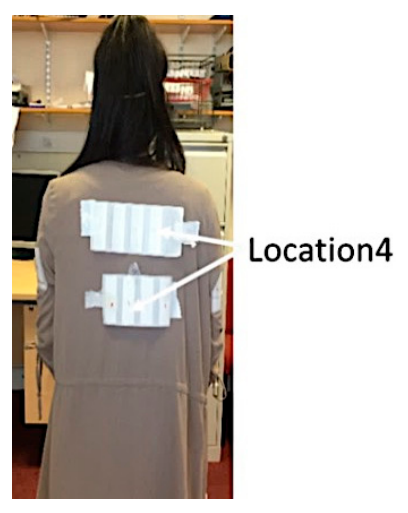

(b)

Figure 1. (a) Front and (b) back view of tag placement on target in a standing position.

Moreover, Figure 2 illustrates the six basic activities of daily living positions in the present experiment. Five positions including standing, walking, sitting, resting and lying in bed, which relative health indicators and fall, which are identified as common causes of domestic accidents are sampled.

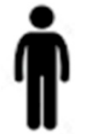

(a)

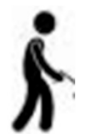

(b)

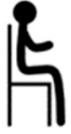

(c)

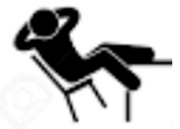

(d)

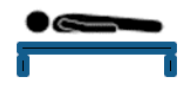

(e)

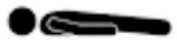

(f)

$\begin{array}{lllll}\text { Standing } & \text { Walking } & \text { Sitting } & \text { Resting }\end{array}$

Figure 2. Six basic activities of daily living patient positions to reflect daily activity.

\subsection{Model Development}

To develop an activity database using the RSSI of passive tags for various positioning, the RSSI model was formulated. Since the patient localisation was RFID-based, with a direct line-of-sight (LOS) between the tags and the RFID reader, a free-space propagation was assumed. Therefore, the RSSI model is expressed in the form:

$$
\operatorname{RSSI}(P)=a \log \delta+b
$$

where $\delta$ is the distance between the tags and the reader, $a$ and $b$ are the model parameters and RSSI (P) is the signal strength indicator measured as the tags RSSI.

Equation (1) considers the effect of multipath as noise, which is influenced by various indoor parameters surrounding the tag and the reader. Moreover, the measurements were of the type $\left\{\left(R S S I_{i, t}, \log \delta_{i, t}\right)\right\}$. Therefore, the objective was to dynamically obtain an RSSI model for each tag, $i$, attached to the body of the patient at a time, $t$. Therefore, taking Equation (1) as a linear function of RSSI and $\log \delta$, the activity database was constructed by performing simple linear least square regression as:

$$
\begin{gathered}
a=\frac{\sum_{t} R S S I_{i, t} \log \delta_{i, t}-\overline{R S S I_{i}} \sum_{t} \log \delta_{i, t}}{\sum_{t}\left(\log \delta_{i, t}\right)^{2}-\overline{\log \delta} \sum_{t} \log \delta_{i, t}} \\
b=\overline{R S S I_{i}}-\left(a_{i} \overline{\delta_{i}}\right)
\end{gathered}
$$

where $\overline{\operatorname{RSSI}}_{i}$ and $\bar{\delta}_{i}$ represents the mean of RSSI and $\delta_{i, t}$ respectively.

An RSS fingerprinting technique using Euclidean distance was employed to develop the offline and online activity database for patient tracking. The Euclidean distance was employed since the 
obtained data varies on more than one feature, i.e., the RSS and location. The error in the model was computed by comparing the position of the tags at each location on the patient using their RSS and the location predicted by the RSSI model. The error in the RSSI model is expressed in the form:

$$
\operatorname{RSSI}_{(\text {error })}=\frac{1}{N} \sum_{t=1}^{M} e_{i, t}^{2}
$$

where

$$
e_{i, t}^{2}=\frac{\left(R S S I_{i, t}-b_{i}\right)}{a_{i}}-\left\|X_{t}-Y_{i}\right\|
$$

From Equation (5), $X_{t}$ represents the patient location at time $t$ and $Y_{t}$ represents the distinct location of each RFID tag.

\subsection{Proof of Concept}

The RSS fingerprinting method was performed through two distinct stages: the offline and the online stage. To illustrate the concept of RF-fingerprinting using the RSS of passive RFID, a key activity for all volunteer was considered. The offline mode is used to capture reference data sets for the known six patient positions. To achieve higher accuracy, two types of averaging were carried out; temporal averaging, where data samples were taken over a period of time and then averaged and spatial averaging, where several tags were mounted on different locations on the patients due to RSS variations. These averaging techniques help reduce the multipath effect on the power level of the received signal. In the offline mode, the dataset from the seven tag locations from six volunteers is highlighted in Figure 3. The sampled dataset is averaged to ameliorate fast fading effect. The sampled dataset was used to develop a reference vector of seven RSS datasets corresponding to each of the six patient positions. The sampled features in each dataset include a timestamp, RSSI and power $(\mathrm{dBm})$ of the RFID tag. Table 2 highlights the averaged reference RSS datasets for the walking position (Walking_Ref) for the six volunteers.

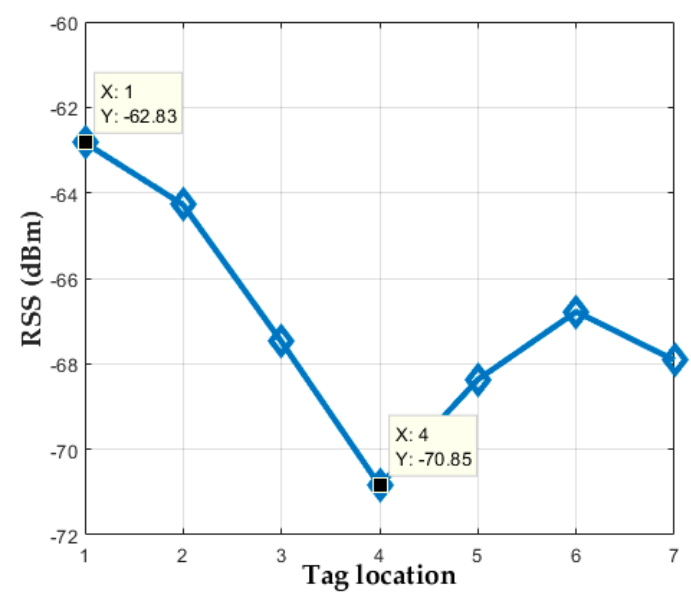

Figure 3. RSS distribution for all seven tag locations on the patient body for walking position.

Table 2. Received signal strength (RSS) values for the Walking Ref position.

\begin{tabular}{cccccccc}
\hline Activity(Walking) & Head & Arm & Waist & Back & Thigh & Knee & Ankle \\
\hline RSS $(\mathrm{dBm})$ & -62.8 & -64.3 & -67.5 & -70.9 & -68.4 & -66.8 & -67.9 \\
\hline
\end{tabular}

Table 2 highlights the computed RSS readings for walking for all six volunteers. It can be seen that the tag location_4 (i.e., back) has the highest RSS which indicates NLOS between the tags and the 
RFID readers. Tag location_1 (head) readily possess the lowest RSS as there is increased LOS between the tags and the RFID reader, as shown in Figure 3.

The maximum RSS variation for all tag locations is in the range of $8.1 \mathrm{dBm}$, which is indicative of the degree of NLOS and occlusion, especially from the furthest tag locations to the RFID readers. However, in the online mode, newly sampled RSS dataset for each for the six test patient positions was captured, analysed via MATLAB and compared with reference position database to validate the accuracy of the system to recognise each position in the testing dataset. The comparison technique employed is the Euclidean distance between testing and reference datasets. From existing works, it is established that the use of Euclidean distance is efficient in mapping an unknown characteristic to one of many stored known characteristics in RF-based systems [34]. Therefore, the Euclidean distance was used to quantitative map the unknown test position dataset with the most probable reference position dataset.

\section{Measurement and Discussion}

Three sets of testing datasets were sampled carried out under LOS and NLOS propagation condition to validate the robustness of the system. Firstly, known stationary positions were used as test position data and mapped against all six positions in the reference database. The same procedure was repeated for all other ADL positions. Table 3 illustrates the distance results of two stationary position of sitting and laying on the floor positions against all six reference positions. From Table 3, it can be seen that the lowest RSSIs are recorded for both correct positions, which validates the robustness of the proposed system for tracking multiple positions.

Table 3. Euclidean distance for "Sitting on the chair" and "Laying on the floor" of patients with the reference position database.

\begin{tabular}{ccccccc}
\hline Test/Ref Positions & $\begin{array}{c}\text { Walking } \\
\text { Reference }\end{array}$ & $\begin{array}{c}\text { Standing } \\
\text { Reference }\end{array}$ & $\begin{array}{c}\text { Sitting } \\
\text { Reference }\end{array}$ & $\begin{array}{c}\text { Resting } \\
\text { Reference }\end{array}$ & $\begin{array}{c}\text { Laying on Floor } \\
\text { Reference }\end{array}$ & $\begin{array}{c}\text { Laying in Bed } \\
\text { Reference }\end{array}$ \\
\hline Sitting Test & 2.4 & 4.0 & 1.4 & 1.4 & 2.9 & 2.5 \\
Laying on Floor Test & 3.2 & 4.7 & 3.3 & 3.7 & 2.1 & 3.1 \\
\hline
\end{tabular}

Moreover, six real-life static and dynamic positions including walking, standing, sitting, resting and laying on the floor were sampled iteratively. Each test dataset was collected over a time period of $15 \mathrm{~s}$ for each sampled iteration. The sampled test dataset was used to estimate the Euclidean distance between the test data and each of the reference position database shown in Figure 4.

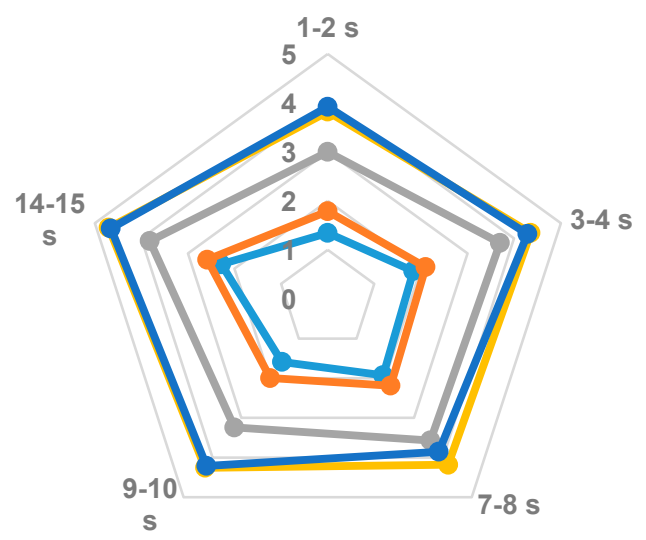

\section{Euclidean RSSI Distance (dB)}

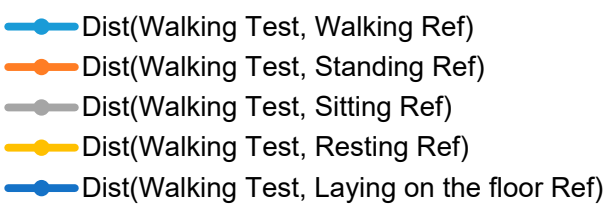

Figure 4. System performance for walking position within the measured time. 
Figure 4 shows that the proposed system accurately identifies walking as the smallest distance in all time periods, indicated by the outer polygon. It is worth noting that since the walking speed of the patient is relatively high compared to standing in the position, the RSSI variation for walking and standing is $3 \mathrm{~dB}$ which shows good correlation. Moreover, seven iterative samplings of standing position of 7s in each sampled iteration are recorded. The Euclidean distance for the standing activity is performed as presented in Figure 5. In Figure 5, it is shown that the standing position exhibits the lowest RSSI of $0.5 \mathrm{~dB}$, which indicate the nearest true position detected. Moreover, it can be seen that the second nearest position is inferred by the proposed system is walking.
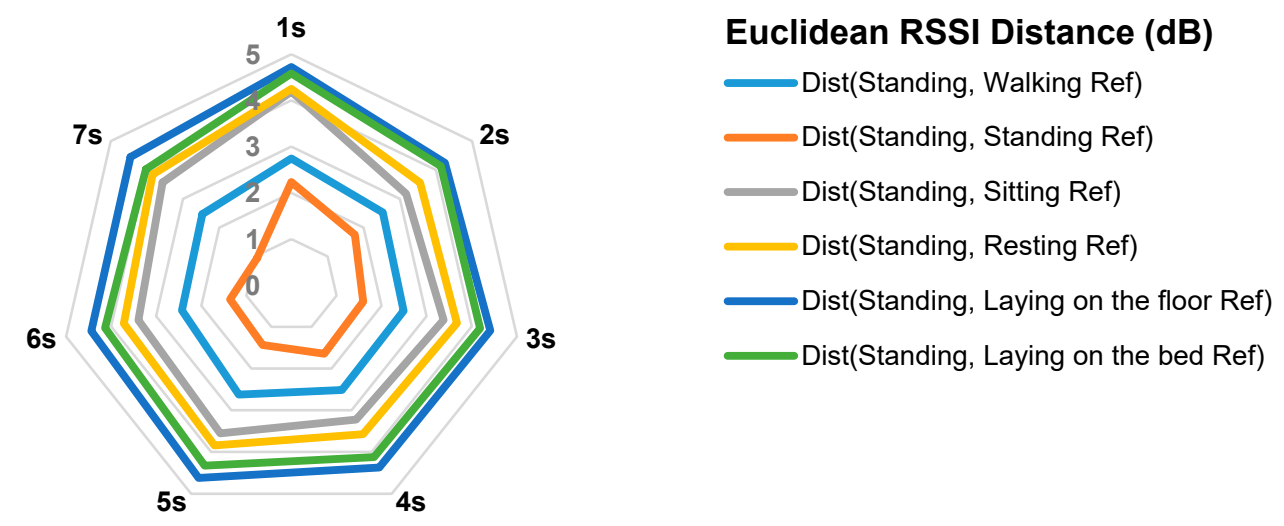

Figure 5. System performance for standing over a stipulated timeframe.

Similar to the standing position, the accuracy of the proposed system is tested for the sitting position using a sampling timeframe of 7s. The result of the sitting position is presented in Figure 6. In Figure 6, the system recognises the correct position as sitting for all time frames. However, it is interesting to observe that the next nearest position detected is resting. This is due to the fact that the tag readings for sitting and resting positions are quite similar.

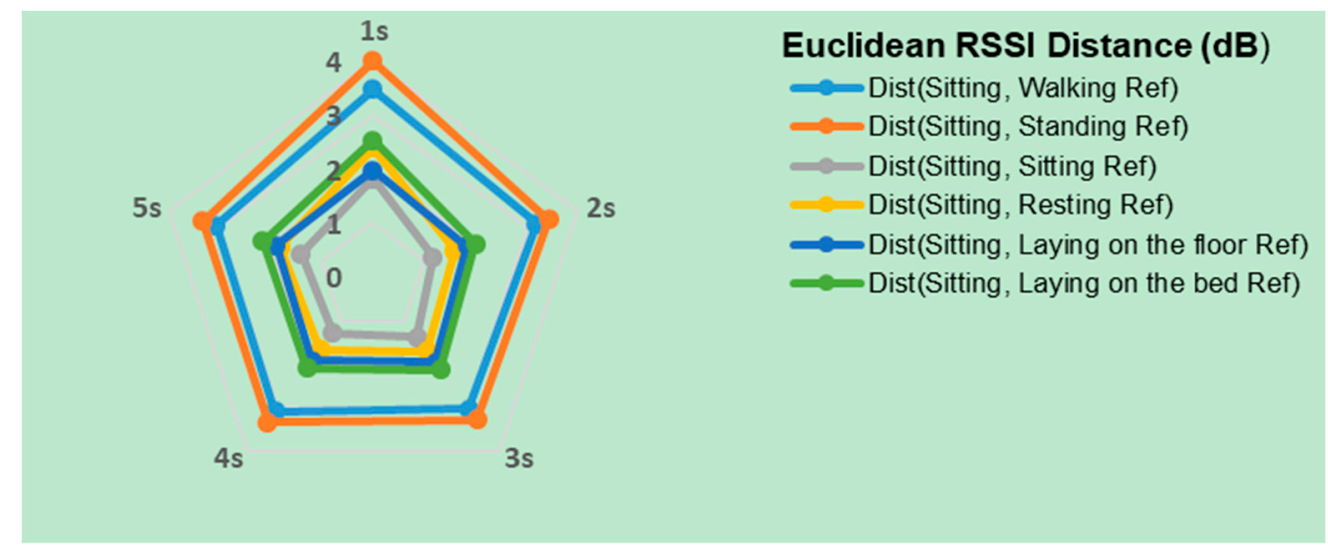

Figure 6. System performance for sitting position over a stipulated timeframe.

It worth noting that fall is a common cause of domestic accidents. To this end, the volunteers are sampled for laying on the fall. The obtained results are compared with the reference database. Figure 7 illustrates the result for the patient laying on the floor for a given period of time. It can be seen from Figure 7 that the true position of each patient is inferred accurately. However, the next closest reference position is sitting with an RSSI of $0.5 \mathrm{~dB}$. 


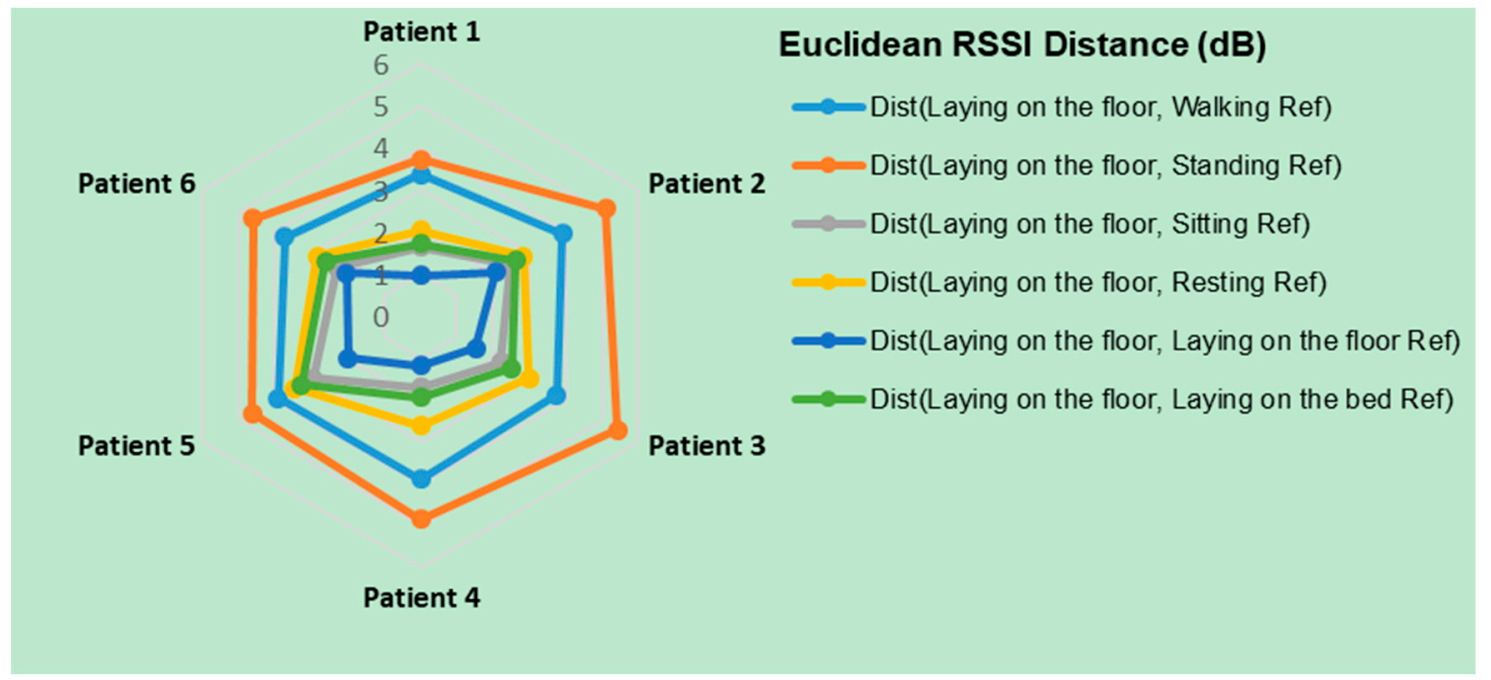

Figure 7. System performance for laying on the floor position over a stipulated timeframe.

Figure 8 illustrates the measured results when a patient lies on the bed for a timeframe of $4 \mathrm{~s}$. The system accurately detects the correct patient position. However, the next nearest position inferred is sitting on the chair with an RSSI of $1.5 \mathrm{dBm}$ whilst the farthest is standing as standing followed by walking.

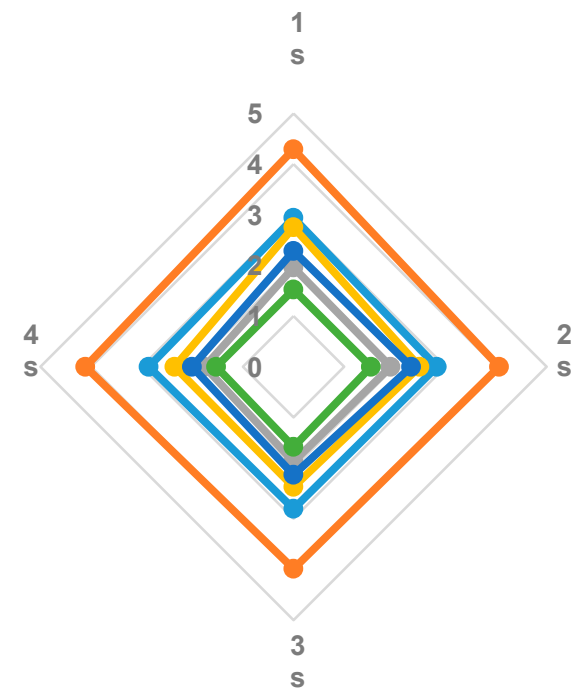

\section{Euclidean RSSI Distance (dB)}

$\longrightarrow$ Dist(Laying on the bed, Walking Ref)

Dist(Laying on the bed, Standing Ref)

$\longrightarrow$ Dist(Laying on the bed, Sitting Ref)

Dist(Laying on the bed, Resting Ref)

$\longrightarrow$ Dist(Laying on the bed, Laying on the floor Ref)

Dist(Laying on the bed, Laying on the bed Ref)

Figure 8. System performance for laying on bed position over a stipulated timeframe.

Furthermore, another case scenario of concurrent position, that is, when targets make different physical movements concurrently over time. Under such condition, the patient does not remain in the same position but changes the activity position with time throughout the entire sampled timeframe as illustrated in Figure 9.

Figure 9 illustrates different human activities with concurrent changes in the target for standing $1 \mathrm{~s}$, sitting for $2 \mathrm{~s}$, walking for another $2 \mathrm{~s}$ and falling whilst remaining in the position for the next $4 \mathrm{~s}$. The result of these scenarios is illustrated in Figure 10. From Figure 10, it can be seen that the system accurately infers the true position at each activity phase based on the smallest distance calculated in comparison with all the other referenced positions. 


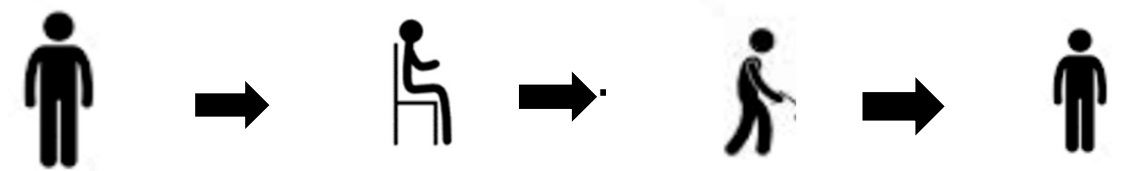

Standing for $1 \mathrm{~s}$

Sitting for $2 \mathrm{~s}$

Walking for $2 \mathrm{~s}$

Laying on the floor for $4 \mathrm{~s}$

Figure 9. Different concurrent activity positions over a stipulated time.

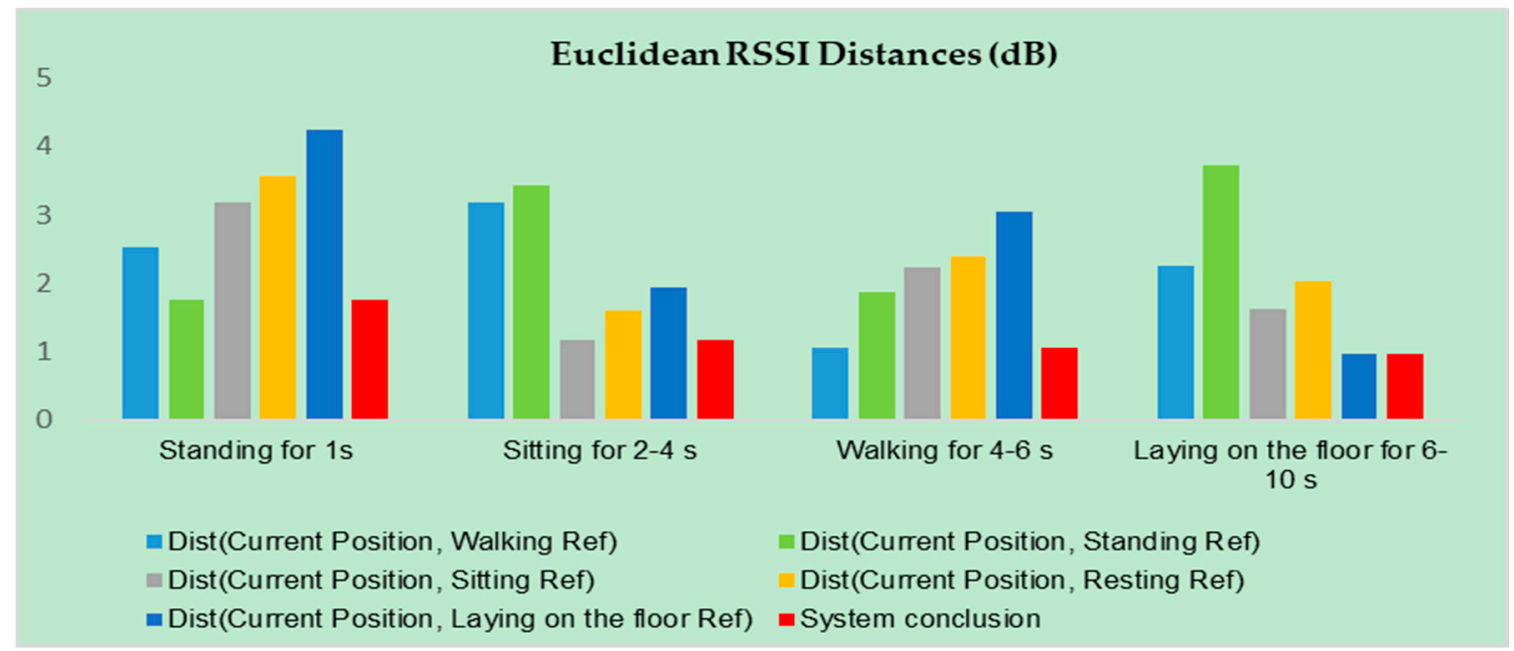

Figure 10. Detection of concurrent target position over a measured time.

\section{Performance Analysis}

The performance of the Euclidean distance model is evaluated using key performance metrics such as precision and recall to validate its robustness for human activity recognition. Precision expresses the ratio of all true positives $(T P)$ to the total predicted positive classes. Precision is usually expressed as:

$$
\text { Precision }=\frac{T P}{T P+F P}
$$

Moreover, recall or sensitivity shows the ratio of accurately predicted positive classes to all activities in the actual class. Recall is expressed as:

$$
\frac{T P}{T P+F N}
$$

From Equations (6) and (7), $F P$, i.e., false-positive refers to all sampled ADLs belonging to other similar (negative) activities inferred as positive categories, and $F N$, i.e., false-negative refers to all true ADL (positive) being classified as negative activities. However, the overall performance of the Euclidean distance model for all sampled ADLs is presented in Table 4.

Table 4. Performance Evaluation of the Euclidean distance model for sampled activities of daily living (ADLs).

\begin{tabular}{ccccccc}
\hline $\begin{array}{c}\text { Performance } \\
\text { Metric }\end{array}$ & Walking & Standing & Sitting & Resting & $\begin{array}{c}\text { Laying on } \\
\text { Floor }\end{array}$ & $\begin{array}{c}\text { Laying in } \\
\text { Bed }\end{array}$ \\
\hline Precision & 0.9801 & 0.9865 & 0.9834 & 0.9831 & 0.9839 & 0.9846 \\
Recall & 0.9773 & 0.9834 & 0.9802 & 0.9800 & 0.9810 & 0.9821 \\
\hline
\end{tabular}




\section{Conclusion}

The development of a cost-effective indoor tracking system using the RSSI of passive RFID tags is presented. RSS fingerprinting using Euclidean distance is used to select the nearest position and compared the reference dataset. The proposed RSS fingerprinting approach using the Euclidean distance model achieves an accuracy of $98 \%$ for most sampled ADLs. However, the accuracy of the activity recognition algorithm performs below the threshold for walking and standing. This is as a result of similarities in target height, weight and body density for these activities. Future work will investigate the 3-D data sampling of these activities and more complex indoor activities to refine the granularity of the data and subsequently the accuracy of the RSS fingerprinting method. The proposed tracking system is applicable to smart health and smart home and is useful to enhance the automated alarming system in nursing homes, as the target stationary position over an extended period of time would be inferred as inactivity such as fall and triggers the alarming system for prompt emergency services.

Author Contributions: W.S., G.O. conceptualise the idea. W.S., G.O., H.O., A.A., R.A. and M.S.B.-M. perform with the experimental measurements. G.O. developed the manuscript. R.A.A.-A., C.K.-Z. and J.N. provide supervisory assistance and in the development of the MATLAB code. All authors have read and agreed to the published version of the manuscript.

Funding: H2020 Marie Skłodowska-Curie Actions: 722424.

Acknowledgments: This work is partially supported by innovation programme under grant agreement H2020-MSCA-ITN-2016 SECRET-722424 and the financial support from the UK Engineering and Physical Sciences Research Council (EPSRC) under grant EP/E022936/1.

Conflicts of Interest: The authors declare no conflicts of interest.

\section{References}

1. United Nations. World Population Ageing 2013; Department of Economic and Social Affairs, Population Division; United Nations: New York, NY, USA, 2013.

2. U. Nations. World Population Ageing 2015; United Nations: New York, NY, USA, 2015; (ST/ESA/SER.A/390).

3. Naughton, C.; Bennett, K.; Feely, J. Prevalence of chronic disease in the elderly based on a national pharmacy claims database. Age Ageing 2006, 35, 633-636. [CrossRef] [PubMed]

4. Ross, C.L. Integral healthcare: the benefits and challenges of integrating complementary and alternative medicine with conventional healthcare practice. Integr. Med. Insights 2009, 4, 13-20. [CrossRef] [PubMed]

5. Biswas, D.; Kristiansen, M.; Krasnik, A.; Norredam, M. Access to healthcare and alternative health-seeking strategies among undocumented migrants in Denmark. BMC Public Health 2011, 11, 560. [CrossRef] [PubMed]

6. Cook, D.J.; Song, W. Ambient Intelligence and Wearable Computing: Sensors on the Body, in the Home, and Beyond. J. Ambient Intell. Smart Environ. 2009, 1, 83-86. [CrossRef] [PubMed]

7. Hänsel, K. Wearable and ambient sensing for well-being and emotional awareness in the smart workplace. In Proceedings of the 2016 ACM International Joint Conference on Pervasive and Ubiquitous Computing Adjunct, Heidelberg, Germany, 12-16 September 2016.

8. Islam, M.S.; Hasan, M.M.; Wang, X.; Germack, H.D.; Noor-E-Alam, M. A Systematic Review on Healthcare Analytics: Application and Theoretical Perspective of Data Mining. Healthcare 2018, 6, 54. [CrossRef] [PubMed]

9. Oguntala, G.A.; Abd-Alhameed, R.A.; Ali, N.T.; Hu, Y.F.; Noras, J.M.; Eya, N.N.; Elfergani, I.; Rodriguez, J. SmartWall: Novel RFID-Enabled Ambient Human Activity Recognition Using Machine Learning for Unobtrusive Health Monitoring. IEEE Access 2019, 7, 68022-68033. [CrossRef]

10. Camci, F. Change Point Detection in Time Series Data using Support Vectors. Int. J. Pattern Recognit. Artif. Intell. 2010, 24, 73-95. [CrossRef]

11. Oguntala, G.; Abd-Alhameed, R.; Jones, S.; Noras, J.; Patwary, M.; Rodriguez, J. Indoor location identification technologies for real-time IoT-based applications: An inclusive survey. Comput. Sci. Rev. 2018, 30, 55-79. [CrossRef] 
12. Ahmad, I.; Asif, R.; Abd-Alhameed, R.A.; Alhassan, H.; Elmegri, F.; Noras, J.M.; See, C.H.; Obidat, H.; Shuaieb, W.; Riberio, J.C.; et al. Current technologies and location-based services. In Proceedings of the 2017 Internet Technologies and Applications (ITA), Wrexham, UK, 12-15 September 2017; pp. 299-304.

13. Wan, L.; Han, G.; Shu, L.; Chan, S.; Zhu, T. The Application of DOA Estimation Approach in Patient Tracking Systems with High Patient Density. IEEE Trans. Ind. Inform. 2016, 12, 2353-2364. [CrossRef]

14. Khoshhal, K.; Aliakbarpour, H.; Quintas, J.; Drews, P.; Dias, J. Probabilistic LMA-based classification of human behaviour understanding using Power Spectrum technique. In Proceedings of the Information Fusion (FUSION), Edinburgh, UK, 26-29 July 2010; pp. 1-7.

15. Yin, J.; Zhang, Q.; Karunanithi, M. Unsupervised daily routine and activity discovery in smart homes. In Proceedings of the 2015 37th Annual International Conference of the IEEE Engineering in Medicine and Biology Society (EMBC), Milan, Italy, 25-29 August 2015; pp. 5497-5500.

16. Bustamante, N.G.P.; Solas, G.; Bilbao, U. In-bed Patients Behaviour Monitoring System. In Proceedings of the Biocomputation, Bioinformatics, and Biomedical Technologies, Bucharest, Romania, 29 June-5 July 2008; pp. 1-6.

17. Motoi, K.; Ogawa, M.; Ueno, H.; Fukunaga, S.; Yuji, T.; Higashi, Y.; Tanaka, S.; Fujimoto, T.; Asanoi, H.; Yamakoshi, K.I. Development and clinical evaluation of a home healthcare system measuring in the toilet, bathtub and bed without attachment of any biological sensors. In Proceedings of the 10th IEEE International Conference on Information Technology and Applications in Biomedicine, Corfu, Greece, 3-5 November 2010; pp. 1-4.

18. Alahmadi, A.; Soh, B. A smart approach towards a mobile e-health monitoring system architecture. In Proceedings of the 2011 International Conference on Research and Innovation in Information Systems, Kuala Lumpur, Malaysia, 23-24 November 2011; pp. 1-5.

19. Reilent, E.; Lõõbas, I.; Pahtma, R.; Kuusik, A. Medical and context data acquisition system for patient home monitoring. In Proceedings of the 2010 12th Biennial Baltic Electronics Conference, Tallinn, Estonia, 4-6 October 2010; pp. 269-272.

20. Kyriazakos, S.; Prasad, N. Delivery of eHealth and eInclusion services for elderly people with mild dementia. In Proceedings of the Wireless Communication, Vehicular Technology, Information Theory and Aerospace \& Electronic Systems Technology (Wireless VITAE), Chennai, India, 28 February-3 March 2011; pp. 1-4.

21. Ayase, T.H.R.; Takayama, S.; Sagawa, S.; Ashida, N. A method for supporting at-home fitness exercise guidance and at-home nursing care for the elders, video-based simple measurement system. In Proceedings of the e-health Networking, Applications and Services, Singapore, Singapore, 7-9 July 2008; pp. 182-186.

22. Long, X.; Gu, I.Y.H.; Flisberg, A.; Thordstein, M. Video-based tracking and quantified assessment of spontaneous limb movements in neonates. In Proceedings of the 2015 17th International Conference on E-health Networking, Application \& Services (HealthCom), Boston, MA, USA, 14-17 October 2015; pp. 517-522.

23. Cuppens, L.L.K.; Ceulemans, B.; van Huffel, S.; Vanrumste, B. Automatic video detection of body movement during sleep based on optical flow in pediatric patients with epilepsy. Med. Biol. Eng. Comput. 2010, 48, 923-931. [CrossRef] [PubMed]

24. Lawrence, E.; Sax, C.; Navarro, K.F.; Qiao, M. Interactive Games to Improve Quality of Life for the Elderly: Towards Integration into a WSN Monitoring System. In Proceedings of the eHealth, Telemedicine, and Social Medicine, Sint Maarten, The Netherlands, 10-16 February 2010; pp. 106-112.

25. Oguntala, G.A.; Abd-Alhameed, R.A.; Jones, S.M.R.; Noras, J.M. Unobtrusive mobile approach to patient location and orientation recognition for elderly care homes. In Proceedings of the 2017 13th International Wireless Communications and Mobile Computing Conference (IWCMC), Valencia, Spain, 26-30 June 2017; pp. 1517-1521.

26. Oguntala, G.; Obeidat, H.; Al Khambashi, M.; Elmegri, F.; Abd-Alhameed, R.A.; Yuxiang, T.; Noras, J.J. Design framework for unobtrusive patient location recognition using passive RFID and particle filtering. In Proceedings of the 2017 Internet Technologies and Applications, ITA, Wrexham, UK, 12-15 September 2017; pp. 212-217.

27. Heikkilä, T.; Strömmer, E.; Kivikunnas, S.; Järviluoma, M.; Korkalainen, M.; Kyllönen, V.; Sarjanoja, E.M.; Peltomaa, I. Low intrusive Ehealth monitoring: human posture and activity level detection with an intelligent furniture network. IEEE Wirel. Commun. 2013, 20, 57-63. [CrossRef] 
28. Freitas, D.J.; Marcondes, T.B.; Nakamura, L.H.V.; Meneguette, R.I. A Health Smart Home System to Report Incidents for Disabled People. In Proceedings of the 2015 International Conference on Distributed Computing in Sensor Systems, Fortaleza, Brazil, 10-12 June 2015; pp. 210-211.

29. Booranrom, Y.; Watanapa, B.; Mongkolnam, P. Smart bedroom for elderly using Kinect. In Proceedings of the Computer Science and Engineering Conference (ICSEC), Khon Kaen, Thailand, 30 July-1 August 2014; pp. 427-432.

30. Martin, E.; Shia, V.; Yan, P.; Kuryloski, P.; Seto, E.; Ekambaram, V.; Bajcsy, R. Enhancing context awareness with activity recognition and radio fingerprinting. In Proceedings of the 2011 IEEE Fifth International Conference on Semantic Computing, Palo Alto, CA, USA, 18-21 September 2011; pp. 263-266.

31. Kim, Y.; Chon, Y.; Cha, H. Smartphone-Based Collaborative and Autonomous Radio Fingerprinting. IEEE Trans. Syst. Man Cybern. Part C (Appl. Rev.) 2012, 42, 112-122. [CrossRef]

32. Jingjing, W.; Xiulong, L.; Wei, S.; Qiuna, N.; Gulliver, T.A.; Xing, L. Fingerprinting localization based on 60 GHz impulse radio. In Proceedings of the 2015 IEEE Pacific Rim Conference on Communications, Computers and Signal Processing (PACRIM), Victoria, BC, Canada, 24-26 August 2015; pp. 491-495.

33. Dama, Y.A.; Abd-Alhameed, R.A.; Hammad, H.; Zaid, R.; Excell, P.S. A Comparison between Vector Algorithm and CRSS Algorithms for Indoor Localization using Received Signal Strength. In Proceedings of the 9th IET International Conference on Computation in Electromagnetics (CEM 2014), London, UK, 31 March-1 April 2014.

34. Bshara, M.; Orguner, U.; Gustafsson, F.; Biesen, L.V. Fingerprinting Localization in Wireless Networks Based on Received-Signal-Strength Measurements: A Case Study on WiMAX Networks. IEEE Trans. Veh. Technol. 2010, 59, 283-294. [CrossRef]

(C) 2020 by the authors. Licensee MDPI, Basel, Switzerland. This article is an open access article distributed under the terms and conditions of the Creative Commons Attribution (CC BY) license (http://creativecommons.org/licenses/by/4.0/). 